\title{
Working
}

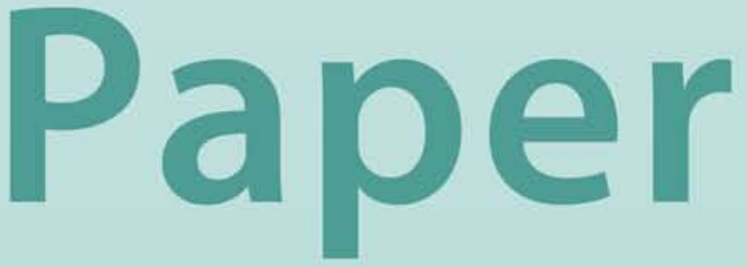


Conformism and Public News

Gabriel Desgranges and Céline Rochon 


\title{
IMF Working Paper
}

IMF Institute

\section{Conformism and Public News}

Prepared by Gabriel Desgranges and Céline Rochon ${ }^{1}$

\author{
Authorized for distribution by Marc Quintyn
}

February 2011

\begin{abstract}
We study a model where investment decisions are based on investors' information about the unknown and endogenous return of the investment. The information of investors consists of endogenously determined messages sold by financial analysts who have access to both public and private information on the return of the investment. We assume that the return of the investment is correlated with the aggregate investment. This results into a beauty contest among analysts (or a “conformism” effect). In equilibrium, analysts sell all the information they have to all the investors. A striking result is that there are sometimes multiple equilibria. There are equilibria where the beauty contest is exacerbated. Because of the correlation across analysts' information sources, not all the information available in the economy is transmitted to investors.
\end{abstract}

JEL Classification Numbers: D82, D84, G14

Keywords: Public Information, Asymmetric Information, Conformism, Beauty Contest

Author’s E-Mail Address: gabriel.desgranges@u-cergy.fr; crochon@imf.org

\section{This Working Paper should not be reported as representing the views of the IMF.} The views expressed in this Working Paper are those of the author(s) and do not necessarily represent those of the IMF or IMF policy. Working Papers describe research in progress by the author(s) and are published to elicit comments and to further debate.

\footnotetext{
${ }^{1}$ THEMA, University of Cergy-Pontoise, France (Desgranges) and IMF and Said Business School, University of Oxford (Rochon). Contact email: gabriel.desgranges@u-cergy.fr; crochon@imf.org.
} 


\section{Contents}

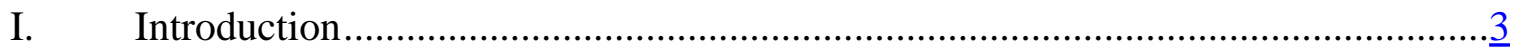

II. The Model ...............................................................................................

III. The Equilibrium ...................................................................................

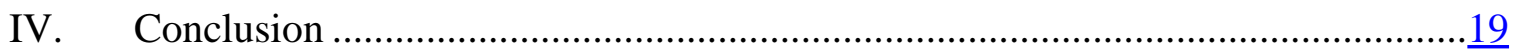

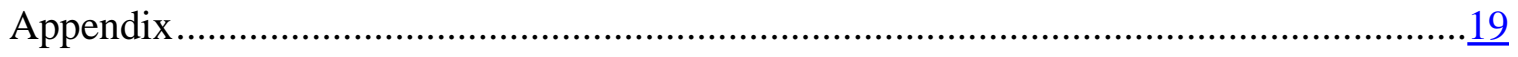

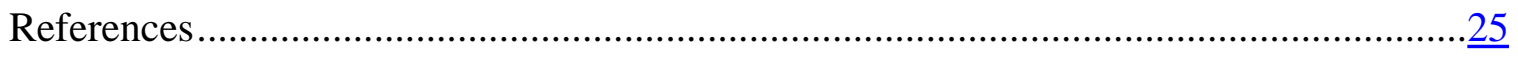




\section{INTRODUCTION}

We develop a model where investors make investment decisions based on information bought from financial analysts about the return of the investment.

The model consists of two building blocks: (i) Analysts determining messages (to be sold to investors) based on their public and private information; (ii) a game between informed investors making investment decisions. An investor's information consists of the messages bought from analysts.

A crucial assumption in this model is that the unknown and endogenous return of the investment is correlated with the aggregate investment. This assumption drives the key mechanism at work in our model: this correlation generates a beauty contest among analysts. Indeed, analysts sell to investors information on the return of the investment. The aggregate investment, which is endogenous, depends on investors' information, namely the messages sent by analysts. Consequently, to predict the return on the investment, an analyst needs to guess current investors' information that correspond to the messages of the other analysts.

Financial analysts hold information in the form of two signals, one privately observed, and one commonly observed by all the analysts. These signals are unknown to the investors, to illustrate the fact that information takes time to be processed and only the analysts possess the expertise to process it. The private signal of an analyst corresponds to its privileged information and his estimation of the impact, on the return, of the information that are commonly observed but whose interpretations differ across analysts. The public signal represents the information that is commonly observed by all analysts and identically interpreted by all analysts.

Financial analysts offer messages to investors based on their private and public signals. Their objective is to maximize their profit.

The objective of investors is to maximize their expected final wealth. It consists of the total net value of the investment and the cost of acquiring messages. Investors' information on the return does not consist of exogenous signals but of endogenously determined messages bought from financial analysts.

We restrict attention to linear and symmetric equilibrium. An equilibrium combines the following 4 properties:

1. The amount invested by investor $i$ is linear in messages and it does not depend on the prices of the messages.

2. Every message is sold to all the investors.

3. Every analyst sells all of his information. 
4. The price of a message is the maximum price that an investor is willing to pay, given his equilibrium information set.

The equilibrium message of an analyst is a linear combination of his public and private signals. We show that the return equation leads to a beauty contest among analysts: The return estimated by an analyst is a linear combination of his prediction of a fundamental and his prediction of other analysts' behavior.

The equilibrium behavior of an analyst involves two features: to give a good prediction about the fundamental, and to conform to the other analysts' predictions. The weight on the average message can be interpreted as a degree of "conformism". This "conformism" is rational and follows from informational motives. It involves both an exogenous and an endogenous component. The exogenous component is related with the correlation between the return and the aggregate investment, while the endogenous component is related with the precision of the messages sold by analysts.

\section{Results.}

- A surprising result is that there are sometimes multiple equilibria. We show that it follows from the endogeneity of the "degree of conformism", which represents the magnitude of the beauty contest.

- There are either one or three linear symmetric equilibria. If the correlation parameter tends to 0 , there is a unique equilibrium. The degree of conformism of this equilibrium tends to 0 . In the limit, this case corresponds to a model where the return is exogenous, and there is no beauty contest.

- When the correlation parameter tends to 1, there are 3 equilibria. Furthermore, the degree of conformism of the 3 equilibria tends to infinity.

- The multiplicity of equilibria leads to two different patterns of behavior. Some equilibria correspond to the standard "beauty contest" result à la Allen, Morris and Shin (2006) where the weight on public information in the message is excessive in order to provide information on others' messages. Other equilibria can be qualified as "inverted" and the effect of the beauty contest is exacerbated. These equilibria display a self-fulfilling misinterpretation of the public signal and overconfidence in private information. By "misinterpretation of the public signal", we mean the following: a high public signal $a$ priori corresponds to a high return of investment; ${ }^{4}$ still the messages sold by analysts to investors are negatively correlated to the public signal. This follows from the endogeneity of the correlation between the messages and the return of investment: this correlation can be strong enough for the equilibrium return to be negatively correlated with the public signal. The negative correlation between messages and public signal is then self-fulfilling.

\footnotetext{
${ }^{4}$ Precisely, the public signal is positively correlated with the exogenous component of the return of investment.
} 
- The observed multiplicity of equilibria can be interpreted as the fact that for some values of the fundamentals, "conformism", or said differently, the magnitude of the beauty contest, is very strong. Analysts can coordinate on different interpretations of the signals, and each of these interpretations has such an important influence on the return of the investment that it is self-fulfilling. Analysts exert "collective manipulation".

- The correlation of the information sources of analysts, and their role in providing information to investors, imply a loss in the information that is transmitted to the investors. This holds true even when each analyst transmits all of his information to all the investors. The messages that an investor buys are correlated. Part of this correlation corresponds to the information conveyed on the fundamental, while the other part of this correlation corresponds to pure noise. The investor is not able to distinguish between these two components of the correlation. This brings about a loss of information. If the sources of information of the analysts were not correlated, this phenomenon would disappear.

Literature. The paper closest in spirit to ours is that of Allen, Morris and Shin (2006). In our paper, as in theirs, the results are driven by an overweighing of the public signal as compared to the private signal. However the overweighing is here motivated by conformism of analysts rather than by the forward-looking structure of an asset pricing model, so that the beauty contest takes place among analysts and not among investors. Furthermore, there is a recent literature dealing with beauty contests, public news and social welfare (Morris and Shin (2002), Hellwig (2005), Angeletos and Pavan (2007) among others). In these papers, there is sometimes a non-monotonic relationship between social welfare and the public information. We offer a model which displays an index of conformism which is endogenous.

Our model is not concerned by usual questions raised by the theoretical literature on financial analysts and investors (Chamley (2003) describes extensively various models). In particular, we do not investigate such issues as reputational effects and verifiability of the analysts' messages (Trueman (1994), Crawford and Sobel (1982), Prendergast (1993), Ottaviani and Sorensen (2001) among others). In our paper, investors rely completely on the messages of the analysts, as only the analysts have the skills to interpret any exogenous signal, private or public. We study the market for information, but not the problem of information acquisition by analysts (Admati and Pfleiderer (1986, 1988, 1990), Barlevy and Veronesi (2000), Verrecchia (1982) among others).

There is a pervasive idea in the literature (Brunnermeier (2001), Chamley (2003), Ottaviani and Sorensen (2000), to name but a few), according to which herding is inefficient and may result in increased volatility. Following the crowd instead of acting on the basis of one's own private information may lead to a loss of information. In a recent paper, Abreu and Brunnermeier (2003) present a model in which the price goes up even though all agents understand that the price is excessively high. These inefficient equilibria rest on the existence of naive traders 
that always follow the crowd. Inefficient equilibria and mispricing are also shown to exist in models where traders care about their reputation for ability (Prat and Dasgupta (2006), Prat, Dasgupta and Verardo (2006)).

There is a large macroeconomic literature considering investment technologies such as the one used in our paper (e.g. Farmer 1999 and the references therein). The investment model used here is an extension to a case with a variable size investment of the well known binary action game where each investor decides either to invest or not invest (the investment size being exogenous) (see Morris 2002 among others). Also, our model is the linear case of a model with strategic complementarities where every agent plays against the average action (with a continuum of agents and a continuous action space). Situations involving strategic complementarities include macroeconomic coordination failures, technology adoption, oligopoly, R\&D competition, to name but a few.

Section 2 presents the model. Section 3 describes the equilibrium. The pricing equation, the beauty contest, and a discussion related to the multiplicity are found in this section. Section 4 concludes. Proofs are gathered in the Appendix.

\section{THE MODEL}

The model consists of two building blocks: (i) a market for information where financial analysts determine the information that is sold to investors; (ii) a game between investors making investment decisions and where the return of investment is endogenous.

The timing of the model is as follows. First, the market for information takes place: investors choose to acquire information (in the form of messages, see below) from analysts on the return $R$ of the investment. Then, investors decide the size of their investment.

$R$ is unknown to investors. Analysts do not know the exact value of $R$, but they have some information regarding $R$, while investors do not.

A crucial assumption is that the return is assumed to be correlated with the aggregate investment, that is:

$$
R=\alpha+\phi X,
$$

where $\phi$ is a real parameter (assume $\phi \in[0,1]$ ), $\alpha$ is an exogenous random variable (a normal variable with mean 0 and precision $\tau_{\alpha}$ ) and $X$ is the aggregate investment. Then, $R$ is determined by an exogenous component $\alpha$ and an endogenous component $X$. This assumption will result into a beauty contest among analysts. Indeed, analysts want to predict $R$. $X$ clearly depends on investors' information, which consists of the messages sent by analysts. Hence, from the viewpoint of an analyst, predicting $R$ requires to predict other analysts' messages. 
This is a beauty contest.

We now describe the model in more details.

The Analysts. There is a finite number $J$ of financial analysts $j$ holding each information on $R$ in the form of two signals correlated with $\alpha$ : a signal $s_{j}=\alpha+\eta_{j}$, and a signal $y=\alpha+\eta_{y}$. Analysts do not observe $X$.

- The signal $s_{j}$ is privately observed by the analyst $j$. All signals have the same precision ( $\eta_{j}$ is a normally distributed variable with mean 0 and precision $\tau_{s}$ ), but every analyst observes a different signal.

- The signal $y$ is commonly observed by the analysts (the value of $y$ is common knowledge among the analysts). $\eta_{y}$ is a normally distributed variable with mean 0 and precision $\tau_{y}$. We call $y$ a public signal for short even if it is not observed by investors.

These signals are unknown to the investors. The use of these two signals by analysts and not by investors translate the fact that information takes time to be processed and requires some specific abilities or training. Analysts have this time and the expertise while investors do not. Along the same lines, the private signal of an analyst (with respect to the public signal) summarizes two kinds of information: the privileged information of the analyst (a genuine private information) and his estimation of the impact on the return of those information pieces that are commonly observed but whose interpretations differ across analysts. In this sense, the so-called public signal $y$ represents the information that is not only commonly observed but also identically interpreted by all analysts, the interpretation being commonly known to be unambiguous.

The random variables $\left(\alpha, \eta_{y}\right.$, and all the $\left.\eta_{j}\right)$ are not correlated. We denote $\bar{\eta}=\sum_{j} \eta_{j} / J$ the average noise. This is a centered normal variable with precision $J \tau_{s}$. We denote $\bar{s}=\alpha+\bar{\eta}$ the average private signal.

Building upon his information, analyst $j$ strategically offers a message $m_{j}$ on $R$ to investors. This message is sold at a price $q_{j}$ to a proportion $N_{j}$ of investors. The objective of the analyst is to maximize his profit. We assume that the production cost of information is zero (an analyst observes $s_{j}$ and $y$ at zero cost) so that the profit is $q_{j} N_{j}$.

A crucial feature of the model is that an analyst offers one message only. This corresponds to the idea that the job of an analyst is to combine all the information sources in a report that is simple enough to be directly exploitable by the investor to make his decision.

This feature of the unique message is crucial because there is no beauty contest without it (as will be made clear later, see in particular the comments of Proposition 4.2). Indeed, if 
an analyst were allowed to sell directly the two pieces of information $y$ and $s_{j}$ instead of a single report $m_{j}$ and if an individual investor had the computational abilities to exploit all these information, then one easily checks that the investor would accept to pay for $y$ and $s_{j}$ (as it would increase his expected profit). In such a model, the role of analysts reduces to the transmission of all their available information without processing it: this boils down to a model without analysts.

The fact that we have only one public signal $y$ and one private signal $s_{j}$ (for each analyst) is w.l.o.g. in a model where all the stochastic variables are normally distributed. Namely, $y$ represents a summary statistic of all the public information and $s_{j}$ represents a summary statistic of all the private information of analyst $j$.

In order to further specify $m_{j}$, we make the following assumption:

Assumption A (normality assumption). The vector $\left(R, y, s_{1}, \ldots, s_{J}, m_{1}, \ldots, m_{J}\right)$ of stochastic variables is a Gaussian vector.

This assumption is made for analytical tractability. It amounts to restricting attention to linear outcomes. Indeed, as is quite common in this type of models, linearity is not a priori obvious. ${ }^{5}$ In particular, for investment decisions to be linear in messages, the information of investors on $R$ must be normally distributed (see below the derivation of the optimal investment decisions). Assumption $\mathrm{A}$ is a simple way to ensure this property.

We elaborate on this in the next section, once the equilibrium concept is defined.

The market for information. The market for information can be described as follows. Analyst $j$ truthfully commits to providing a message $m_{j}$ with given properties (in particular: informativeness on $R$ and covariance with messages of other analysts). That is: an analyst sells to an investor at a price $q_{j}$ a guarantee that he will draw $m_{j}$ from a given announced distribution $\left(m_{j}, s_{j}, y\right)$.

At this stage, each investor does not observe the message yet, but he knows the laws of the messages, allowing him to compute the expected marginal benefit from each message. Each investor decides then how many messages and which ones he buys.

Implicit assumptions in this market for information are: analysts cannot discriminate among investors (analysts cannot sell the same message at different prices to different investors),

\footnotetext{
${ }^{5}$ We do not address the question of non linearity. Considering non linear reports would imply considering non gaussian distributions, and therefore non linear investment decisions, which complicates the model in a non trivial way.
} 
investors do not resell messages to each other, and analysts do not buy messages from other analysts. 6

The investment decision. We turn now attention to the investors' behavior. There is a continuum [0,1] of infinitesimal investors. Every investor makes an investment decision $x_{i} \in \mathbb{R}$ which depends on the agent's information set $I_{i}\left(I_{i}\right.$ is the set of analysts $j$ from which investor $i$ buys a message). A main feature of the model is that investors' information on $R$ does not consist of exogenous signals but of endogenously determined messages bought from financial analysts.

The objective of investors is to maximize the expected final wealth. This final wealth consists of 2 parts: the total net value of the investment $x_{i}$ and the cost of acquiring messages. Assuming that the investment has a return $R$ and an installation cost $\frac{1}{2} x_{i}^{2}$, the final wealth is:

$$
R x_{i}-\frac{1}{2} x_{i}^{2}-\sum_{j \in I_{i}} q_{j}
$$

The maximization problem leads to an optimal investment $x_{i}\left(I_{i}\right)$ and an optimal demand for messages (i.e. the maximum price that $i$ is willing to pay for a message $m_{j}$ ).

We now compute $x_{i}\left(I_{i}\right)$. We provide the optimal demand for messages in the next section.

For a given set of messages $I_{i}, x_{i}\left(I_{i}\right)$ solves

$$
\max _{x_{i}} E\left(R \mid I_{i}\right) x_{i}-\frac{1}{2} x_{i}^{2} .
$$

Then, for a given information set $I_{i}, x_{i}\left(I_{i}\right)$ does not depend on the prices $q_{j}$ paid for the messages (as the expected profit is linear in the prices $q_{j}$ of the messages). Furthermore, $i$ 's investment takes the following simple form: ${ }^{7}$

$$
x_{i}\left(I_{i}\right)=E\left(R \mid I_{i}\right)
$$

Assumption A implies then that $x_{i}\left(I_{i}\right)$ is linear in the messages bought by $i$ from analysts.

We have shown:

- Property 1: The amount $x_{i}\left(I_{i}\right)$ invested by investor $i$ is $E\left(R \mid I_{i}\right)$; it is linear in messages; it does not depend on the prices $q_{j}$ of the messages.

\footnotetext{
${ }^{6}$ Along the same lines, we do not allow analysts to invest.

${ }^{7}$ Because of normal distributions, $x_{i}\left(I_{i}\right)$ can be negative. As usual when normal distributions are used (see Grossman 1976 for an early example of this argument), this should not be seen as a problem: the probability of a negative $x_{i}\left(I_{i}\right)$ can be made arbitrarily small (by an appropriate choice of $E(\alpha)$ and $\tau_{\alpha}$ for example).
} 
The return $R$ is defined by:

$$
R=\alpha+\phi X,
$$

where $X=\int x_{i} d i$ is the aggregate investment. ${ }^{8}$

Some comments about Equation (2) are now in order.

Comment 1. Equation (2) is a reduced form model for an investment game, where the return of an individual investment depends on the aggregate investment level. It embodies the idea that there is a positive externality to investment or, equivalently, the investment technology displays increasing returns to scale at the social level (while, at the individual level, the investment displays constant returns equal to $R$ ). There is a large macroeconomic literature considering such investment technologies (see for example Farmer 1999 and the references therein). Notice also that this investment model is an extension to a case with a variable size investment of the well known binary action game where each investor decides either to invest or not invest (the investment size being exogenous) (see Morris 2002 among others).

Comment 2. The key property of the model under consideration is that the optimal investment writes:

$$
x_{i}\left(I_{i}\right)=E\left(\alpha+\phi X \mid I_{i}\right),
$$

that is: the individual decision $x_{i}$ is the expected value of an exogenous variable $\alpha$ and an aggregate $X$ of others' decisions (linearity makes the model tractable). As will be made clear in the next section, the exogenous uncertain variable $\alpha$ creates the need for expert information provided by analysts. Then, the aggregate $X$ is correlated with individual expert information, which creates the beauty contest among analysts (as investors require information not only on $\alpha$ but on $(\alpha+\phi X))$.

Comment 3. The above "investors" story is only one of the possible stories associated with Equation (3). More generally, from an abstract viewpoint, our model is the linear case of a model with strategic complementarities where every agent plays against the mean action (with a continuum of agents and a continuous action space). There are many situations involving strategic complementarities (macroeconomic coordination failures, technology adoption, oligopoly, R\&D competition,...). ${ }^{9}$

Simple models that generate the best-response map (3) include:

- Price-setting monopolistic competition: each agent $i$ is a monopolistic producer setting a price $x_{i}$ for its product and facing a demand $\left(\alpha-\frac{1}{2} x_{i}+\phi X\right)$ where $X$ is now interpreted

\footnotetext{
$8 \int x_{i} d i$ is the standard notation in a world with a continuum of investors. A rigorous writing will be provided in a next Section.

${ }^{9}$ see the seminal paper by Milgrom and Roberts (1990) for a survey of applications.
} 
as a price index (the goods produced by others are substitutes of $i$ 's production as $\phi>0$ ). For a given information set $I_{i}$, the expected profit of $i$ writes $E\left(\alpha-\frac{1}{2} x_{i}+\phi X \mid I_{i}\right) x_{i}$ (there is no production cost). Equation (3) results.

- Quantity-setting monopolistic competition: the same story as above but $x_{i}$ is interpreted as the quantity produced and $\left(\alpha-\frac{1}{2} x_{i}+\phi X\right)$ as an inverse demand function (the goods produced by others are complements of $i$ 's production as $\phi>0)$.

- A speculative attack model: the speculator $i$ invests an amount $x_{i}$ in attacking a peg, and the return on the attack (the magnitude of the devaluation) is increasing in the aggregate amount $X$ invested in the attack.

Notice that we assume $\phi>0$, but a negative $\phi$ would not destroy our results. ${ }^{10}$ If we allow $\phi$ to be negative, then the reduced form model includes the monopolistic competition models even if goods are complements (in the price-setting case) or substitutes (in the quantity-setting case). The model includes also the partial equilibrium model where agents produce all the same good and each agent $i$ makes a production decision $x_{i}$ before he knows the price (and the price is $\alpha+\phi X$ with $\phi<0$ ) (see among others Muth 1961, Guesnerie 1992, Morris 2008).

\section{THE EQUILIBRIUM}

We are now in a position to define the equilibrium concept. The natural equilibrium concept is the perfect Bayesian equilibrium involving out of equilibrium beliefs. ${ }^{11}$ An exhaustive definition of this equilibrium concept would be tedious. To avoid it, we restrict attention to linear and symmetric equilibrium, and we present 3 properties (in addition to Property 1 above). These properties unquestionably hold at a linear symmetric equilibrium, whatever the formal definition of equilibrium may be. This allows us to give then a simplified formal definition of the equilibrium.

- Property 2: Every message $m_{j}$ is sold to all the investors.

Symmetry of equilibrium implies that a message $m_{j}$ is bought either by no investor or all of them. As the production cost of $m_{j}$ by analyst $j$ is zero, it is always profitable for analyst $j$ to sell $m_{j}$ at a positive price, even arbitrarily small. This implies that, at equilibrium, every message $m_{j}$ is sold to all the investors.

\footnotetext{
${ }^{10}$ In particular, our central result (Proposition 3.4) does not disappear: there are still cases with multiple equilibria when $\phi<0$. But the computations when $\phi<0$ are more sophisticated.

${ }^{11}$ To see why out-of-equilibrium beliefs are in order, consider that one of the problems faced by an analyst is to choose the message he sells to investors. In order to know what the profit maximizing message is, the analyst has to expect what price investors are ready to pay for any message he could offer, and not only for the one message that he offers at equilibrium.
} 
- Property 3: Every analyst sells all of his information. That is: the distribution of $R$ conditional on $m_{j}$ is the distribution of $R$ conditional on $\left(s_{j}, y\right)$.

The production cost of a message is zero and the analyst wants to maximize his profit. As the willingness to pay of an investor is increasing in the precision of the message, the analyst thus sells all the information he has in order to charge a price $q_{j}$ as large as possible. This follows from the benefit of a piece of information for an investor being increasing in the precision of this piece of information.

The proof of this property is in Appendix. To prove this property, we assume that an individual analyst considers as exogenous the distribution of return. Analysts have a "return-taking" behavior.

Assumption B. Every analyst $j$ considers the joint distribution of $\left(R, s_{j}, y\right)$ as exogenous.

Assumption B implies that an analyst does not understand that the choice of his message influences investors' decisions and then aggregate investment and return. Hence, no analyst tries to manipulate investor's decisions and the return $R$; each analyst only aims at guessing $R$ as if $R$ did not depend on his own message. This amounts to assuming that each analyst regards as negligible the influence of his own advice on the investors' decisions. This assumption seems legitimate in the case of a large number $J$ of analysts. ${ }^{12}$

- Property 4: The price $q_{j}$ of message $m_{j}$ is the maximum price that an investor is willing to pay, given his equilibrium information set $I_{i}$.

Given the symmetry of equilibrium, the maximal price that an investor $i$ is willing to pay does not depend on $i$. The demand faced by analyst $j$ for $m_{j}$ is such that every investor buys $m_{j}$ if $q_{j}$ is smaller than the common maximal price, and no investor buys $m_{j}$ otherwise. Then, the profit maximizing analyst $j$ charges this common maximal price for $m_{j}$.

Relying on the 4 properties above, we can define formally the equilibrium message and its associated price.

According to Assumption A and Property $3, m_{j}$ can be written as a linear combination of $y$ and $s_{j}$. We choose to normalize the weights on $y$ and $s_{j}$ so that they sum to 1 . That is: we define $m_{j}$ as follows:

\footnotetext{
${ }^{12}$ Actually, relaxing Assumption B allows to consider the case where an analyst strategically takes into account the impact of his message on $R$. But (even in the case of 2 analysts only), this leads to a model that is not tractable (the equilibrium message is characterized as the root of a polynomial of degree 6 ).
} 
Definition 3.1 The equilibrium message $m_{j}$ writes:

$$
\forall j, m_{j}=\mu y+(1-\mu) s_{j}
$$

where $\mu$ is a real parameter independent of $j$.

The fact that $\mu$ does not depend on $j$ follows from the symmetry of equilibrium.

The fact that the weights $\mu$ and $(1-\mu)$ sum to 1 is w.l.o.g. because the informational content of $m_{j}$ is the same as the one of $k m_{j}$ for any real coefficient $k$ : the law of $R$ conditional to $k m_{j}$ is not affected by $k$. Notice that $m_{j}$ is proportional to $E\left(R \mid s_{j}, y\right)\left(m_{j}\right.$ is $k E\left(R \mid s_{j}, y\right)$ where the coefficient of proportionality $k$ is such that the weights on $s_{j}$ and $y$ sum to 1 ). We could have defined $m_{j}$ as $E\left(R \mid s_{j}, y\right)$, but Definition 3.1 implies that $m_{j}$ writes $\alpha+$ noise, which simplifies computations later. We have namely:

$$
m_{j}=\alpha+\mu \eta_{y}+(1-\mu) \eta_{j}
$$

We characterize the equilibrium value of $\mu$ in the next section.

Lemma 3.2. The price $q_{j}$ of message $m_{j}$ is

$$
q_{j}=\frac{1}{2}\left(\operatorname{Var}\left(R \mid m_{1}, \ldots, m_{j-1}, m_{j+1}, \ldots, m_{J}\right)-\operatorname{Var}\left(R \mid m_{1}, \ldots, m_{J}\right)\right),
$$

where $\operatorname{Var}\left(R \mid m_{1}, \ldots, m_{j-1}, m_{j+1}, \ldots, m_{J}\right)$ and $\operatorname{Var}\left(R \mid m_{1}, \ldots, m_{J}\right)$ are variances of $R$ conditional on the sets $\left\{m_{1}, \ldots, m_{J}\right\}-\left\{m_{j}\right\}$ and $\left\{m_{1}, \ldots, m_{J}\right\}$ respectively.

The expression of $q_{j}$ given in Equation (5) is obtained from the following argument. The maximum price that investor $i$ is willing to pay for $m_{j}$ is the price that makes $i$ indifferent between buying $m_{j}$ or not. In Appendix, we show that the ex-ante expected profit of an investor $i$ buying a set of messages $I_{i}$ is (see Equation (14) in Appendix): ${ }^{13}$

$$
E \pi\left(I_{i}\right)=\frac{1}{2}\left(\operatorname{Var}(R)-\operatorname{Var}\left(R \mid I_{i}\right)\right)-\sum_{j \in I_{i}} q_{j} .
$$

At equilibrium, $i$ buys all the messages so that his ex-ante expected profit is $E \pi\left(\left\{m_{1}, \ldots, m_{J}\right\}\right)$. If he decides not to buy $m_{j}$, then his ex-ante expected profit becomes $E \pi\left(\left\{m_{1}, \ldots, m_{J}\right\}-\left\{m_{j}\right\}\right)$. Equating the 2 expected profits leads to Equation (5).

Summing up, we have the following equilibrium definition. It is clear from this definition that an equilibrium is fully characterized by the value of $\mu$.

\footnotetext{
${ }^{13}$ Notice that, as every investor is infinitesimal, the equilibrium distribution of $R$ does not depend on individual choices by investors.
} 
Definition 3.3. Under Assumption A, a symmetric linear equilibrium is defined by the following behavior of agents:

- Investor $i$ buys all the messages $m_{j}$ and his investment is

$$
E\left(R \mid m_{1}, \ldots, m_{J}\right)
$$

- Analyst $j$ sells the message

$$
m_{j}=\mu y+(1-\mu) s_{j}
$$

at price

$$
q_{j}=\frac{1}{2}\left(\operatorname{Var}\left(R \mid m_{1}, \ldots, m_{j-1}, m_{j+1}, \ldots, m_{J}\right)-\operatorname{Var}\left(R \mid m_{1}, \ldots, m_{J}\right)\right)
$$

The Return Equation. A standard property of Gaussian distributions is that the average message $\bar{m}=\sum_{j} m_{j} / J$ is a summary statistic of $\left(m_{1}, \ldots, m_{J}\right)$, that is: the distribution of $R$ conditional on $\left(m_{1}, \ldots, m_{J}\right)$ is the same as the distribution of $R$ conditional on $\bar{m}$. The investment of $i$ is then

$$
E(R \mid \bar{m}),
$$

aggregate investment is $E(R \mid \bar{m})$ as well, and the return equation (2) writes:

$$
R=\alpha+\phi E(R \mid \bar{m})
$$

Investors' information is symmetric so that $E(R \mid \bar{m})$ is the belief about $R$ common to all investors.

The Beauty Contest. The return equation (6) naturally leads to a description of the beauty contest among analysts. Taking the expected values (conditional on $\bar{m}$ ) on both sides in Equation (6) imply: ${ }^{14}$

$$
\begin{aligned}
E(R \mid \bar{m}) & =E(\alpha \mid \bar{m})+\phi E(R \mid \bar{m}) \\
& =\frac{1}{1-\phi} E(\alpha \mid \bar{m}) \\
& =\frac{1}{1-\phi} \frac{\operatorname{cov}(\alpha, \bar{m})}{\operatorname{Var}(\bar{m})} \bar{m} .
\end{aligned}
$$

Hence, $R$ is a linear function of $\bar{m}$ and $\alpha$

$$
R=\alpha+\lambda \bar{m}
$$

${ }^{14}$ Recall the standard formula for conditional means of normally distributed variables:

$$
E(Z \mid \bar{m})=E(Z)+\frac{\operatorname{cov}(Z, \bar{m})}{\operatorname{Var}(\bar{m})}(\bar{m}-E(\bar{m})) .
$$


where $\lambda$ is endogenous and defined by

$$
\lambda=\frac{\phi}{1-\phi} \frac{\operatorname{cov}(\alpha, \bar{m})}{\operatorname{Var}(\bar{m})}=\frac{\phi}{1-\phi} \frac{\frac{1}{\tau_{\alpha}}}{\frac{1}{\tau_{\alpha}}+\mu^{2} \frac{1}{\tau_{y}}+(1-\mu)^{2} \frac{1}{J \tau_{s}}} .
$$

From Equation (7), we have:

$$
\forall j, E\left(R \mid s_{j}, y\right)=E\left(\alpha \mid s_{j}, y\right)+\lambda E\left(\bar{m} \mid s_{j}, y\right) .
$$

This relation shows that analysts implicitly participate in a beauty contest (a guess-the-average game): the return $R$ estimated by analyst $j$ (and then the message sent by $j$ ) is a linear combination of his prediction of a fundamental $\alpha$ and his prediction of others' behavior $\bar{m}$.

The intuition for this beauty contest is as follows:

- The aggregate investment $X$ aggregates the information used by investors when making their investment decision $x_{i}$ (this information consists of the messages $m_{j}$ ).

- The assumption that $R$ is correlated with $X$ implies that $R$ is correlated with the messages $m_{j} .{ }^{15}$

- Investors have no prior information on $R$ so that they pay analysts to make predictions about $R$. It follows that every analyst needs to predict other analysts' messages.

Equation (9) shows that the behavior of the analyst involves two features: to give a good prediction about $\alpha$, or else to conform to the other analysts' predictions and hence not to distinguish oneself too much from the group of analysts. The weight $\lambda$ on $E\left(\bar{m} \mid s_{j}, y\right)$ can be interpreted as a degree of "conformism". This "conformism" is rational and follows from informational motives. The degree of conformism involves two components: an exogenous component $\frac{\phi}{1-\phi}$ and an endogenous component $\frac{\operatorname{cov}(\alpha, \bar{m})}{\operatorname{Var}(\bar{m})}$.

In the sequel, we focus on the determination of conformism, its influence on the information transmitted by analysts to the market, and its consequences on the market return.

Solving for the equilibrium message. We now compute the equilibrium message, that is: we write $\bar{m}=\mu y+(1-\mu) \bar{s}$ in Equation (9)

$$
\forall j, E\left(R \mid s_{j}, y\right)=\lambda E\left(\mu y+(1-\mu) \bar{s} \mid s_{j}, y\right)+E\left(\alpha \mid s_{j}, y\right) .
$$

This writes $E\left(R \mid s_{j}, y\right)=\mu^{\prime} y+\nu^{\prime} s_{j}$ where $\mu^{\prime}$ and $\nu^{\prime}$ are 2 real coefficients (to be computed in the proof of the Proposition below). As $m_{j}=\mu y+(1-\mu) s_{j}$, we have $m_{j}=\frac{\mu^{\prime} y+\nu^{\prime} s_{j}}{\mu^{\prime}+\nu^{\prime}}$.

\footnotetext{
${ }^{15}$ By symmetry of the equilibrium, the correlation between $R$ and the messages $m_{j}$ reduces to a correlation between $R$ and $\bar{m}$.
} 
The equilibrium values of $\mu$ are the solutions of the equation $\mu=\frac{\mu^{\prime}}{\mu^{\prime}+\nu^{\prime}}$. A striking result is that there are sometimes multiple equilibria. It follows from the endogeneity of the so-called "degree of conformism" $\lambda$ (that is: the magnitude of the beauty contest).

Proposition 3.4. Assume $\phi \in[0,1]$. There exists a threshold $\phi^{*}$ such that

1. If $\phi>\phi^{*}$, then there are 3 linear symmetric equilibria.

2. Otherwise, $\phi<\phi^{*}$, and there is 1 linear symmetric equilibrium.

One equilibrium is always characterized by a value $\mu \in\left[\frac{\tau_{y}}{\tau_{s}+\tau_{y}}, 1\right]$, while the two others (when they exist) are such that $\mu \leq-\frac{\tau_{y}}{\tau_{\alpha}}$.

The threshold $\phi^{*}$ is

$$
\phi^{*}=\frac{1}{\frac{J-1}{J} A^{*}+1} \in(0,1)
$$

where

$$
A^{*}=\sup _{\mu \leq-\tau_{y} / \tau_{\alpha}} \frac{(\mu-1)\left(\tau_{y}+\mu \tau_{\alpha}\right)}{\left(\tau_{y}-\left(\tau_{s}+\tau_{y}\right) \mu\right)\left(1+\mu^{2} \frac{\tau_{\alpha}}{\tau_{y}}+(1-\mu)^{2} \frac{\tau_{\alpha}}{J \tau_{s}}\right)} \in(0,+\infty) .
$$

The proof of the Proposition can be found in Appendix. It relies on solving a polynomial equation of degree 3 in $\mu$. This is why there exists either 1 or 3 equilibria. $^{16}$

The proposition shows that there is a unique equilibrium when $\phi$ is around 0 and there is multiplicity when $\phi$ is around 1. Before we comment on the proposition, we further describe the 2 examples $\phi=0$ and $\phi=1$.

\section{Corollary 3.5.}

- When $\phi$ tends to 0, there is a unique equilibrium. Furthermore, the degree of conformism $\lambda$ of this equilibrium tends to 0 ; the equilibrium value of $\mu$ tends to $\frac{\tau_{y}}{\tau_{y}+\tau_{s}}$.

- When $\phi$ tends to 1 , there are 3 equilibria. Furthermore, the degree of conformism $\lambda$ of the 3 equilibria tends to $+\infty$; one equilibrium value of $\mu$ tends to 1 , another tends to $-\tau_{y} / \tau_{\alpha}$, and the third one tends to $-\infty$.

In the limit $\phi=0$, we have the case where $R=\alpha$ is exogenous. Every analyst simply sends a message revealing $E\left(\alpha \mid s_{j}, y\right)$, and there is no beauty contest.

\footnotetext{
${ }^{16}$ The limit case $\phi=1$ corresponds to a degenerate situation where the polynomial equation of degree 3 has 3 real roots, two of them being identical.
} 
In the limit case $\phi=1$, the model is not determined (Equation (6) above has no solution). The proposition nevertheless shows that, when $\phi \simeq 1$, the multiplicity of equilibria is robust and the equilibria correspond to an exacerbated beauty contest (the degree of conformism $\lambda$ becomes infinite). This appears clearly for the equilibrium with $\mu \simeq 1$ (the messages rely on the public signal only and no longer on the private signal). The two other equilibria feature a negative $\mu$. Further interpretations of the equilibria with $\mu<0$ are provided in the comments below.

Comments are in order regarding the intuition for the multiplicity and the behavioral patterns at equilibrium.

Comment 1: Intuition for the existence of multiple equilibria. The main reason for the observed multiplicity is that, for some values of the fundamentals $\left(\phi, \tau_{y}, \tau_{s}\right.$ and $\left.\tau_{\alpha}\right)$, the "conformism" $\lambda$ (in other words, the magnitude of the beauty contest) can be so large that analysts "collectively manipulate" the prices. The correlation between $X$ and $R$ along with the influence the analysts have on $X$ through their messages (recall $X=E(R \mid \bar{m})$ ) allow them to move $R$ in any direction as soon as all their individual messages are well coordinated. Indeed, at an equilibrium, analysts coordinate their interpretation of exogenous signals (they choose an equilibrium value of $\mu$ ). Multiplicity of equilibria means that they can coordinate on different interpretations of the signals, and each of these interpretations has such an important influence on $R$ that it is self-fulfilling.

Comment 2: equilibrium patterns. The multiplicity of equilibria corresponds to 2 different patterns of behavior. Some equilibria have $\mu \in[0,1]$ and can be qualified as "regular". These correspond to the standard "beauty contest" result à la Allen, Morris and Shin (2006) where the weight on public information in the message is excessive in order to provide information on others' messages.

Other equilibria have $\mu<0$ and can be qualified as "inverted". The effect of the beauty contest is then exacerbated. In contrast with the "regular" equilibrium, these "inverted" equilibria feature 2 appealing properties. These properties are interpreted as follows:

- Self-fulfilling misinterpretation of the public signal. $\mu<0$ means that every message $m_{j}$ reacts negatively to the public signal. This can be understood as a collective voluntary misinterpretation of $y$ as every analyst is aware that $y$ and $\alpha$ are positively correlated. Still, the coordination motive is so strong that this misinterpretation of $y$ is self-fulfilling: $y$ is negatively correlated with $R$ at equilibrium.

- Overconfidence in private information. The weight $(1-\mu)$ on $s_{j}$ in the message is larger than 1. This translates the idea that the analyst has chosen an "inverted" weight on $y$ to inform on $\bar{m}$, but this results in false information conveyed on $\alpha$. This is compensated by an "overconfidence" in the private signal. 
An "inverted" equilibrium can be thought of as a "bubble" story. For example, when $y$ is largely negative, every analyst agrees on public information revealing future bad market fundamentals. But, it is commonly known that this bad public signal translates into a global recommendation to invest (that is: positive messages $m_{j}$ ). As this "invest" recommendation is believed by investors, it leads to an increase in the aggregate investment $X$. This increase in $X$ is expected to result in an increase in the return (as $R$ is expected to be $\alpha+\phi X$ ). It follows that analysts are right in recommending investors to invest: analysts' and investors' behavior is an equilibrium behavior.

Precision of information transmitted by analysts. We want to consider the precision of information transmitted to the investors by analysts. Two approaches are possible. The first one is to consider the precision of the information on the fundamentals $\alpha$ sent by analysts to investors. This precision has an exogenous upper bound (which is the precision of information available to analysts on $\alpha$ ). The second approach is to consider the precision of the information given on $R$. This is relevant as $R$ is the variable in investors' objective.

But Equation (7) above (that is: $R=\alpha+\lambda \bar{m}$ ) clearly shows that

$$
\operatorname{Var}(R \mid \bar{m})=\operatorname{Var}(\alpha \mid \bar{m}) \text {. }
$$

It follows that the 2 approaches defined above are identical.

The information available in the economy about $\alpha$ consists of the public signal $y$ and the collection of the private signals $s_{j}$. The precision of this information is

$$
\tau_{\alpha \mid y, s_{1}, \ldots, s_{J}}=\tau_{\alpha \mid y, \bar{s}}=\tau_{\alpha}+\tau_{y}+J \tau_{s}
$$

We can compare this precision to $\tau_{\alpha \mid \bar{m}}=1 / \operatorname{Var}(\alpha \mid \bar{m})$. Thus, we can unambiguously state whether the analysts transmit to investors all the available information in the economy. We show that this is not the case: although each analyst individually sends all his information to all the investors, the "beauty contest" effect implies that there is a loss in the aggregate information transmitted to investors.

Proposition 3.6. Denote $\tau_{\alpha \mid y, s_{1}, \ldots, s_{J}}$ the precision of the information available in the economy about $\alpha$. Denote $\tau_{\alpha \mid m_{1}, \ldots, m_{J}}$ the precision of the information transmitted to each investor by analysts through the messages $\left(m_{1}, \ldots, m_{J}\right)$. We have:

$$
\tau_{\alpha \mid m_{1}, \ldots, m_{J}}<\tau_{\alpha \mid y, s_{1}, \ldots, s_{J}} .
$$

Not all the information available on $\alpha$ is transmitted to investors.

The proof of Proposition 4.2 is purely computational. It is in Appendix. 
Because of correlation of the information sources of analysts, the very existence of analysts implies a loss in the information that is transmitted to the market. This holds true even in the case under consideration here where each analyst transmits all of his information to all the investors. The messages that an investor buys are correlated. Part of this correlation corresponds to the information conveyed on $\alpha$ (or, equivalently, $R$ ), the other part of this correlation corresponds to pure noise. The investor is not able to distinguish between these two components of the correlation. This is the reason why some information loss occurs. If analysts' information sources were not correlated (conditionally to $\alpha$ ), ${ }^{17}$ then this phenomenon would disappear, and the above inequality (11) would be an equality.

\section{CONCLUSION}

We have considered a simple model where the return of individual investment is increasing in the aggregate investment. The specific feature of our model is that the private information of investors is endogenous: it consists of messages sent by profit maximizing analysts. These messages are determined by analysts on the basis of their information.

This results in a beauty contest among analysts: every analyst needs to predict others' messages to correctly predict the return of investment. Our main result is that there is sometimes multiple equilibria. One equilibrium is a "regular" beauty contest where analysts overweight the public signal in the messages they send to investors. Another, more surprising, equilibrium is an "inverted" equilibrium where analysts put a negative weight on the public signal: the coordination motive among analysts is so strong that it is self-fulfilling for analysts to commonly misinterpret the public signal (that is: to expect a high public signal to imply a low return).

\section{APPENDIX}

Proof of Property 3. We show that the ex-ante profit of investor $i$ is increasing in the precision of his information.

Denote $I_{i}$ the set of $j$ such that investor $i$ buys the message from analyst $j$. We compute the ex-ante profit of an investor $i$ buying a set of messages $m_{j}, j \in I_{i}$.

For a given set $I_{i}$ and given messages $m_{j}$

$$
\pi\left(\left(m_{j}\right)_{j \in I_{i}}\right)=\max _{x_{i}} E\left(R \mid\left(m_{j}\right)_{j \in I_{i}}\right) x_{i}-\frac{x_{i}^{2}}{2}-\sum_{j \in I_{i}} q_{j}
$$

The optimal investment is $x_{i}=E\left(R \mid\left(m_{j}\right)_{j \in I_{i}}\right)$ and the expected profit conditional to the

\footnotetext{
${ }^{17}$ This would be the case in a model with no public signal $y$. Each analyst would transmit his private signal to investors, and each investor would observe all the information available to analysts.
} 
messages $m_{j}$ is

$$
\pi\left(\left(m_{j}\right)_{j \in I_{i}}\right)=\frac{1}{2} E\left(R \mid\left(m_{j}\right)_{j \in I_{i}}\right)^{2}-\sum_{j \in I_{i}} q_{j} .
$$

For a given set $I_{i}$, the ex-ante expected profit is then

$$
E \pi\left(I_{i}\right)=E\left[\pi\left(\left(m_{j}\right)_{j \in I_{i}}\right)\right]=\frac{1}{2} E\left[E\left(R \mid\left(m_{j}\right)_{j \in I_{i}}\right)^{2}\right]-\sum_{j \in I_{i}} q_{j},
$$

where the mean $E[$.$] is taken over the values of the messages m_{j}, j \in I_{i}$. As long as $R$ and the $m_{j}$ are centered normally distributed (which will be shown to be true at equilibrium), a summary statistic for a vector of messages $\left(m_{j}\right)_{j \in I_{i}}$ consists of one message $\hat{m}$ only (a linear combination of $\left.m_{j}\right)$ and $E\left(R \mid\left(m_{j}\right)_{j \in I_{i}}\right)$ writes

$$
E\left(R \mid\left(m_{j}\right)_{j \in I_{i}}\right)=\frac{\operatorname{cov}(R, \hat{m})}{\operatorname{Var}(\hat{m})} \hat{m} .
$$

Hence, we have:

$$
E\left[E\left(R \mid\left(m_{j}\right)_{j \in I_{i}}\right)^{2}\right]=\left(\frac{\operatorname{cov}(R, \hat{m})}{\operatorname{Var}(\hat{m})}\right)^{2} E\left(\hat{m}^{2}\right)=\frac{\operatorname{cov}(R, \hat{m})^{2}}{\operatorname{Var}(\hat{m})} .
$$

Recall the usual formula for the conditional variance of Gaussian variables:

$$
\operatorname{Var}(R \mid \hat{m})=\operatorname{Var}(R)-\frac{\operatorname{cov}(R, \hat{m})^{2}}{\operatorname{Var}(\hat{m})} \text {. }
$$

It follows that:

$$
E\left[E\left(R \mid\left(m_{j}\right)_{j \in I_{i}}\right)^{2}\right]=\operatorname{Var}(R)-\operatorname{Var}(R \mid \hat{m})
$$

As $\hat{m}$ is a summary statistic of $\left(m_{j}\right)_{j \in I_{i}}$, we have $\operatorname{Var}(R \mid \hat{m})=\operatorname{Var}\left(R \mid\left(m_{j}\right)_{j \in I_{i}}\right)$ and Equation (12) writes:

$$
E \pi\left(I_{i}\right)=\frac{1}{2}\left(\operatorname{Var}(R)-\operatorname{Var}\left(R \mid\left(m_{j}\right)_{j \in I_{i}}\right)\right)-\sum_{j \in I_{i}} q_{j} .
$$

The higher the precision of the information $\left(m_{j}\right)_{j \in I_{i}}$ available to investor $i$, the larger the ex-ante profit $E \pi\left(I_{i}\right)$. End of the proof.

Proof of Proposition 3.4. Given $\bar{m}=\mu y+(1-\mu) \bar{s}$, we have:

$$
\begin{aligned}
E\left(\bar{m} \mid s_{j}, y\right) & =E\left(\mu y+(1-\mu) \frac{1}{J} \sum s_{k} \mid s_{j}, y\right) \\
& =\mu y+(1-\mu) \frac{1}{J}\left(s_{j}+E\left(\sum_{k \neq j} s_{k} \mid s_{j}, y\right)\right) \\
& =\mu y+(1-\mu) \frac{1}{J}\left(s_{j}+(J-1) E\left(\alpha \mid s_{j}, y\right)+\sum_{k \neq j} E\left(\eta_{k} \mid s_{j}, y\right)\right), \\
& =\mu y+(1-\mu) \frac{1}{J}\left(s_{j}+(J-1) E\left(\alpha \mid s_{j}, y\right)\right) .
\end{aligned}
$$


Hence, Equation (10) rewrites:

$$
\forall j, E\left(R \mid s_{j}, y\right)=\lambda\left(\mu y+(1-\mu) \frac{1}{J} s_{j}+(1-\mu)(J-1) \frac{1}{J} \frac{\tau_{s} s_{j}+\tau_{y} y}{\tau_{\alpha}+\tau_{s}+\tau_{y}}\right)+\frac{\tau_{s} s_{j}+\tau_{y} y}{\tau_{\alpha}+\tau_{s}+\tau_{y}} .
$$

Write $E\left(R \mid s_{j}, y\right)=\mu^{\prime} y+\nu^{\prime} s_{j}$. We have:

$$
\begin{aligned}
\mu^{\prime} & =\lambda\left(\mu+(1-\mu)(J-1) \frac{1}{J} \frac{\tau_{y}}{\tau_{\alpha}+\tau_{s}+\tau_{y}}\right)+\frac{\tau_{y}}{\tau_{\alpha}+\tau_{s}+\tau_{y}} \\
\nu^{\prime} & =\lambda\left((1-\mu) \frac{1}{J}+(1-\mu)(J-1) \frac{1}{J} \frac{\tau_{s}}{\tau_{\alpha}+\tau_{s}+\tau_{y}}\right)+\frac{\tau_{s}}{\tau_{\alpha}+\tau_{s}+\tau_{y}} .
\end{aligned}
$$

Given that $m_{j}=\mu y+(1-\mu) s_{j}$, we have seen above that an equilibrium value of $\mu$ is characterized by $\mu=\mu^{\prime} /\left(\mu^{\prime}+\nu^{\prime}\right)$. Hence, we have:

$$
\mu=\frac{\lambda\left(\mu+(1-\mu)(J-1) \frac{1}{J} \frac{\tau_{y}}{\tau_{\alpha}+\tau_{s}+\tau_{y}}\right)+\frac{\tau_{y}}{\tau_{\alpha}+\tau_{s}+\tau_{y}}}{\lambda\left(\mu+(1-\mu) \frac{1}{J}+(1-\mu)(J-1) \frac{1}{J} \frac{\tau_{s}+\tau_{y}}{\tau_{\alpha}+\tau_{s}+\tau_{y}}\right)+\frac{\tau_{s}+\tau_{y}}{\tau_{\alpha}+\tau_{s}+\tau_{y}}} .
$$

Some computations allows us to rewrite this equation in the following more convenient form:

$$
\tau_{\alpha} \mu^{2}+\left(\tau_{y}-\tau_{\alpha}\right) \mu-\tau_{y}=\left(\tau_{y}-\left(\tau_{s}+\tau_{y}\right) \mu\right) \frac{J}{J-1} \frac{1}{\lambda}
$$

Recall from Equation (8) that:

$$
\frac{1}{\lambda}=\frac{1-\phi}{\phi}\left(1+\mu^{2} \frac{\tau_{\alpha}}{\tau_{y}}+(1-\mu)^{2} \frac{\tau_{\alpha}}{J \tau_{s}}\right)
$$

Hence $\mu$ is the root of a polynomial of degree 3 :

$$
\tau_{\alpha} \mu^{2}+\left(\tau_{y}-\tau_{\alpha}\right) \mu-\tau_{y}=A\left(\tau_{y}-\left(\tau_{s}+\tau_{y}\right) \mu\right)\left(1+\mu^{2} \frac{\tau_{\alpha}}{\tau_{y}}+(1-\mu)^{2} \frac{\tau_{\alpha}}{J_{\tau_{s}}}\right)
$$

where $A=\frac{J}{J-1} \frac{1-\phi}{\phi}$. Denote

$$
\begin{aligned}
\operatorname{LHS}(\mu) & =\tau_{\alpha} \mu^{2}+\left(\tau_{y}-\tau_{\alpha}\right) \mu-\tau_{y}=(\mu-1)\left(\tau_{y}+\mu \tau_{\alpha}\right) \\
Q(\mu) & =\left(\tau_{y}-\left(\tau_{s}+\tau_{y}\right) \mu\right)\left(1+\mu^{2} \frac{\tau_{\alpha}}{\tau_{y}}+(1-\mu)^{2} \frac{\tau_{\alpha}}{J \tau_{s}}\right) .
\end{aligned}
$$

LHS is a polynomial of degree 2 with roots $-\tau_{y} / \tau_{\alpha}$ and $1 . Q$ is a polynomial of degree 3 with one real root $\tau_{y} /\left(\tau_{s}+\tau_{y}\right)$ only and the coefficient of degree 3 is negative.

Equation (16) writes $\operatorname{LHS}(\mu)=A Q(\mu)$. Notice that

$$
A^{*}=\sup _{\mu \leq-\tau_{y} / \tau_{\alpha}} \frac{\operatorname{LHS}(\mu)}{Q(\mu)} .
$$


The analysis of the solutions of Equation (16) is partitioned in 4 regions of values of $\mu$ corresponding to the 4 following Lemmas.

Lemma A.1. There is no solution of $(16)$ in $\left[-\tau_{y} / \tau_{\alpha}, \tau_{y} /\left(\tau_{s}+\tau_{y}\right)\right]$.

Proof of Lemma A.1. For $\mu$ in $\left[-\tau_{y} / \tau_{\alpha}, 1\right], L H S(\mu)<0$ and for $\mu<\tau_{y} /\left(\tau_{s}+\tau_{y}\right), A Q(\mu)>$ 0 . End of the proof.

Lemma A.2. There is no solution of (16) in $[1,+\infty)$.

Proof of Lemma A.2. For $\mu \geq 1, L H S(\mu) \geq 0$ and for $\mu>\tau_{y} /\left(\tau_{s}+\tau_{y}\right), A Q(\mu)<0$. End of the proof.

Lemma A.3. There is exactly one solution of $(16)$ in $\left[\tau_{y} /\left(\tau_{s}+\tau_{y}\right), 1\right]$.

Proof of Lemma A.3. For $\mu=\tau_{y} /\left(\tau_{s}+\tau_{y}\right)$, LHS $(\mu)-A Q(\mu)<0$. For $\mu=1, \operatorname{LHS}(\mu)-$ $A Q(\mu)>0$. Hence, there is at least one solution of $(16)$ in $\left[\tau_{y} /\left(\tau_{s}+\tau_{y}\right), 1\right]$.

To see that there is only one solution, one computes the inflection point $\mu^{I}$ (the minimum of $\left.Q^{\prime}\right)$. We have:

$$
\begin{aligned}
Q^{\prime}(\mu)= & -\left(\tau_{s}+\tau_{y}\right)\left(1+\mu^{2} \frac{\tau_{\alpha}}{\tau_{y}}+(1-\mu)^{2} \frac{\tau_{\alpha}}{J \tau_{s}}\right) \\
& +2\left(\tau_{y}-\left(\tau_{s}+\tau_{y}\right) \mu\right)\left(\mu \frac{\tau_{\alpha}}{\tau_{y}}-(1-\mu) \frac{\tau_{\alpha}}{J \tau_{s}}\right),
\end{aligned}
$$

and

$$
\begin{aligned}
Q^{\prime \prime}(\mu)= & -2\left(\tau_{s}+\tau_{y}\right)\left(\mu \frac{\tau_{\alpha}}{\tau_{y}}-(1-\mu) \frac{\tau_{\alpha}}{J \tau_{s}}\right) \\
& -2\left(\tau_{s}+\tau_{y}\right)\left(\mu \frac{\tau_{\alpha}}{\tau_{y}}-(1-\mu) \frac{\tau_{\alpha}}{J \tau_{s}}\right) \\
& +2\left(\tau_{y}-\left(\tau_{s}+\tau_{y}\right) \mu\right)\left(\frac{\tau_{\alpha}}{\tau_{y}}+\frac{\tau_{\alpha}}{J \tau_{s}}\right) .
\end{aligned}
$$

$\mu^{I}$ is defined by $Q^{\prime \prime}\left(\mu^{I}\right)=0$. Hence,

$$
\mu^{I}=\frac{\left(2 \tau_{s}+3 \tau_{y}\right) \frac{1}{J \tau_{s}}+1}{3\left(\tau_{s}+\tau_{y}\right)\left(\frac{1}{\tau_{y}}+\frac{1}{J \tau_{s}}\right)}
$$

Some more computations show that (given $J>1$ )

$$
\mu^{I}<\frac{\tau_{y}}{\tau_{s}+\tau_{y}} .
$$


It follows that, for $\mu$ in $\left[\tau_{y} /\left(\tau_{s}+\tau_{y}\right), 1\right], A Q$ is concave. As $L H S$ is convex on this interval, $L H S-A Q$ is convex on this interval as well. This implies that there cannot be more than one root of $L H S-A Q$ in $\left[\tau_{y} /\left(\tau_{s}+\tau_{y}\right), 1\right]$. End of the proof.

Lemma A.4. There exists $A^{*}>0$ such that ${ }^{18}$

- if $A>A^{*}$, then Equation (16) has no solution in $\left(-\infty,-\tau_{y} / \tau_{\alpha}\right]$,

- if $0<A<A^{*}$, then Equation (16) has 2 solutions in $\left(-\infty,-\tau_{y} / \tau_{\alpha}\right]$,

Proof of Lemma A.4. From Equation (17), $Q^{\prime}(\mu)<0$ for $\mu<0$ (the first term of $Q^{\prime}$ is negative, the second term is the product of a positive factor and a negative factor). Hence, for $\mu \leq-\tau_{y} / \tau_{\alpha}, Q(\mu) \geq Q(0)>0$. Recall that for $\mu \leq-\tau_{y} / \tau_{\alpha}, L H S(\mu) \geq 0$. Hence,

$$
\forall \mu \leq-\tau_{y} / \tau_{\alpha}, \frac{\operatorname{LHS}(\mu)}{Q(\mu)} \geq 0
$$

Notice that $\frac{L H S(\mu)}{Q(\mu)}$ tends to 0 when $\mu$ tends to $-\infty$ (as $L H S$ and $Q$ are of degree 2 and 3 respectively). It follows that the range of $\frac{L H S(\mu)}{Q(\mu)}$ when $\mu \in\left(-\infty,-\tau_{y} / \tau_{\alpha}\right]$ is $\left[0, A^{*}\right]$.

Hence, for every $A$ in $\left[0, A^{*}\right]$, there is at least one $\mu \leq-\tau_{y} / \tau_{\alpha}$ such that $A=\frac{L H S(\mu)}{Q(\mu)}$. This value of $\mu$ is a solution of Equation (16). As Equation (16) is a polynomial equation of degree 3 , it admits either 1 or 3 real solutions. The 3 previous lemmas imply then that there is exactly one solution of Equation (16) outside $\left(-\infty,-\tau_{y} / \tau_{\alpha}\right]$. Hence, there must be 2 solutions of Equation $(16)$ in $\left(-\infty,-\tau_{y} / \tau_{\alpha}\right]$.

Lastly, for $A>A^{*}$, there is no $\mu \leq-\tau_{y} / \tau_{\alpha}$ such that $A=\frac{\operatorname{LHS}(\mu)}{Q(\mu)}$. Hence, Equation (16) has no solution in $\left(-\infty,-\tau_{y} / \tau_{\alpha}\right]$ when $A>A^{*}$. End of the proof.

Combining the 4 lemmas shows the proposition. End of the proof.

Proof of Corollary 3.5. When $\phi=0, A=+\infty$ and Equation (16) becomes

$$
0=\left(\tau_{y}-\left(\tau_{s}+\tau_{y}\right) \mu\right)\left(1+\mu^{2} \frac{\tau_{\alpha}}{\tau_{y}}+(1-\mu)^{2} \frac{\tau_{\alpha}}{J \tau_{s}}\right) .
$$

The unique solution is $\mu=\frac{\tau_{y}}{\tau_{y}+\tau_{s}}$. From Equation (8), $\lambda=0$.

When $\phi$ tends to 1, Equation (8) shows $\lambda$ tends to $+\infty$. Furthermore, $A$ tends to 0. As Equation (16) is $\operatorname{LHS}(\mu)=A Q(\mu)$, the RHS tends to 0 when $\phi$ tends to 1 . Then, when $\phi$ tends to 1, it can easily be seen that there is one real root of Equation (16) in the neighborhood of each of the 2 roots of $L H S(\mu)=0$, which are 1 and $-\frac{\tau_{y}}{\tau_{\alpha}} \cdot{ }^{19}$ As Equation (16) is a polynomial equation of degree 3 , there is a third real root. To check that this third root tends to $-\infty$, notice that the product of the 3 roots of Equation (16) tends to infinity (it is well known that this product is the ratio of 2 coefficients of the polynomial). End of the proof.

\footnotetext{
${ }^{18}$ The case $A=A^{*}$ is "degenerate" and leads to a situation where Equation (16) has 1 solution in $\left(-\infty,-\tau_{y} / \tau_{\alpha}\right]$ (the 2 solutions of the case $A<A^{*}$ compress to one).

${ }^{19}$ The roots of a polynomial equation are continuous in the coefficients of the polynomial.
} 
Proof of Proposition 3.6. Standard computations show that

$$
\tau_{\alpha \mid s_{1}, \ldots, s_{J}, y}=\tau_{\alpha \mid \bar{s}, y}=\tau_{\alpha}+\tau_{\bar{s}}+\tau_{y}=\tau_{\alpha}+J \tau_{s}+\tau_{y}
$$

Using the relation $\bar{m}=\mu y+(1-\mu) \bar{s}$, the same standard computations show that

$$
\tau_{\alpha \mid m_{1}, \ldots, m_{J}}=\tau_{\alpha \mid \bar{m}}=\tau_{\alpha}+\frac{1}{\mu^{2} \frac{1}{\tau_{y}}+(1-\mu)^{2} \frac{1}{J \tau_{s}}},
$$

given that $\bar{m}=\alpha+\mu \eta_{y}+(1-\mu) \bar{\eta}$ and the precision of the noise $\mu \eta_{y}+(1-\mu) \bar{\eta}$ is

$$
\frac{1}{\mu^{2} \frac{1}{\tau_{y}}+(1-\mu)^{2} \frac{1}{J \tau_{s}}} .
$$

The inequality between the precisions writes:

$$
\frac{1}{\mu^{2} \frac{1}{\tau_{y}}+(1-\mu)^{2} \frac{1}{J \tau_{s}}}<J \tau_{s}+\tau_{y} .
$$

This rewrites

$$
\begin{aligned}
1 & <\mu^{2}\left(1+\frac{J \tau_{s}}{\tau_{y}}\right)+(1-\mu)^{2}\left(1+\frac{\tau_{y}}{J \tau_{s}}\right) \\
0 & <\mu^{2}\left(1+\frac{J \tau_{s}}{\tau_{y}}+1+\frac{\tau_{y}}{J \tau_{s}}\right)+\frac{\tau_{y}}{J \tau_{s}}-2 \mu\left(1+\frac{\tau_{y}}{J \tau_{s}}\right) \\
0<\mu & \mu^{2} \frac{J \tau_{s}}{\tau_{y}}+(1-\mu)^{2} \frac{\tau_{y}}{J \tau_{s}}-2 \mu(1-\mu) \\
0 & <\left(\mu \sqrt{\frac{J \tau_{s}}{\tau_{y}}}-(1-\mu) \sqrt{\frac{\tau_{y}}{J \tau_{s}}}\right)^{2}
\end{aligned}
$$

Then, the proposition holds true as soon as the above RHS is different from 0 , that is:

$$
\mu \neq \frac{\sqrt{\frac{\tau_{y}}{J \tau_{s}}}}{\sqrt{\frac{\tau_{y}}{J \tau_{s}}}+\sqrt{\frac{J \tau_{s}}{\tau_{y}}}}=\frac{\tau_{y}}{\tau_{y}+J \tau_{s}} .
$$

We check that Equation (16) has no solution equal to $\frac{\tau_{y}}{\tau_{y}+J \tau_{s}}$ when $\phi$ is different from the value specified in the proposition. With $\mu=\frac{\tau_{y}}{\tau_{y}+J \tau_{s}}$, Equation (16) writes:

$$
-J \tau_{s}=A(J-1) \tau_{s}
$$

where $A=\frac{J}{J-1} \frac{1-\phi}{\phi}$. This is impossible. This shows the result. End of the proof. 


\section{REFERENCES}

Abreu D, M. Brunnermeier (2003), Bubbles and crashes. Econometrica 71: 173-204.

Admati A, P. Pfleiderer (1986), A monopolistic market for information. Journal of Economic Theory 39: 400-438.

Admati A, P. Pfleiderer (1988), Selling and trading on information in financial markets. American Economic Review 78: 96-103.

Admati A, P. Pfleiderer (1990), Direct and indirect sale of information. Econometrica 58: 901-928.

Allen F, S. Morris, H. Shin (2006), Beauty contests and iterated expectations in asset markets. The Review of Financial Studies 19: 719-752.

Angeletos, G-M, G. Lorenzoni, A. Pavan (2007), Wall Street and Silicon Valley: a delicate interaction. mimeo.

Angeletos, A. Pavan (2007), Efficient use of information and social value of information. Econometrica 75: 1103-1142.

Barlevy B, P. Veronesi (2000), Information acquisition in financial markets. The Review of Economic Studies 67, 79-90.

Brunnermeier M (2001), Asset pricing under asymmetric information: bubbles, crashes, technical analysis, and herding. Oxford University Press.

Chamley C (2003), Rational herds: economic models of social learning. Cambridge University Press.

Chordia T, R. Roll, A. Subrahmanyam (2001), Market liquidity and trading activity. The Journal of Finance 56: 501-530.

Crawford V, J. Sobel (1982), Strategic information transmission. Econometrica 50: 14311451.

Dasgupta A, A. Prat (2005), Reputation and asset prices: a theory of information cascades and systematic mispricing, mimeo.

Dasgupta A, A. Prat, M. Verardo (2006), The price of conformism, mimeo.

Farmer R. (1999), The macroeconomics of self-fulfilling prophecies. MIT Press. 
Grossman S (1976), On the efficiency of competitive stock markets where trades have diverse information. The Journal of Finance 31: 573-585.

Grossman S, J. Stiglitz (1980), On the impossibility of informationally efficient markets. American Economic Review 70: 393-408.

Guesnerie R. (1992), An exploration of the eductive justifications of the rational-expectations hypothesis. American Economic Review 82(5): 1254-1278.

Hellwig C, (2005), Heterogeneous information and the welfare effects of public information disclosures. mimeo.

Morris, S (2002) Coordination, Communication and Common Knowledge: A Retrospective on the Electronic-Mail Game. Oxford Review of Economic Policy 1: 433-445.

Morris, S, H. Shin (2002), Social value of public information. The American Economic Review 92: 1521-1534.

Muth J.F. (1961), Rational expectations and the theory of price movements. Econometrica 29(3): 315-335.

Ottaviani M, P. Sorensen (2000), Herd behavior and investment: comment. American Economic Review 90: 695-704.

Ottaviani M, P. Sorensen (2001), Information aggregation in debate: who should speak first. Journal of Public Economics 81: 393-421.

Prendergast C (1993), A theory of yes men. American Economic Review 83: 757-770.

Subrahmanyam A (1991), Risk aversion, market liquidity and price efficiency. The Review of Financial Studies 4: 417-441.

Trueman B (1994), Analyst forecasts and herding behavior. The Review of Financial Studies 7: 97-124.

Verrecchia F (1982), Information acquisition in a noisy rational expectations economy. Econometrica 50: 1415-1430. 